\title{
A NOTE ON A THEOREM OF MOSTOW
}

P. E. CONNER

Recently Mostow showed that if the fundamental group of the homogeneous space of a Lie group is solvable, then it is finitely generated and its rank is bounded by the dimension of the homogeneous space [4]. This generalizes the Smith result that the rank of the fundamental group of a Lie group cannot exceed the dimension of the Lie group [6]. Our note is motivated by an attempt to sharpen the upper bound on the rank of the solvable fundamental group. We do this, and in case the homogeneous space is compact with solvable fundamental group, we calculate the rank exactly.

By $\left(\pi, M^{n}\right)$ we shall denote a proper transformation group on a connected manifold [2]. Associated to every proper transformation group $\left(\pi, M^{n}\right)$ is the quotient space $M^{n} / \pi$ and the covering map

$$
\eta: M^{n} \rightarrow M^{n} / \pi \text {. }
$$

There is also a spectral sequence $\left\{E_{r}^{s, t}\right\}$ with

$$
E_{2}^{s, t} \simeq H^{8}\left(\pi ; H^{t}\left(M^{n} ; J\right)\right)
$$

and whose $E_{\infty}$-term is associated with $H^{*}\left(M^{n} / \pi ; J\right)$. The coefficient group $J$ will be either the integers, $Z$, or the integers mod a prime $p$, $Z_{p}$. This spectral sequence (1) is called the spectral sequence of a covering [2].

In case $\pi$ is a finite group, there is another spectral sequence $\left\{\hat{E}_{r}^{s, t}\right\}$ with

$$
\hat{E}_{2}^{s, t} \simeq H^{8}\left(\pi ; H_{c}^{t}\left(M^{n} ; J\right)\right)
$$

and whose $E_{\infty}$-term is associated with $H_{c}^{*}\left(M^{n} / \pi ; J\right)$. The subscript $c$ refers to cohomology based on cochains with compact support [1]. We shall use the maximal and the minimal cocycle arguments on these two spectral sequences [2].

THEOREM 1. Let $\left(\pi, M^{n}\right)$ denote an abelian group of rank $m$ acting as a proper group of transformations on a manifold $M^{n}$. If $H^{i}\left(M^{n} ; Z\right)$ $=0$ for $i>n-m$, then one of the following three alternatives holds

1. $H^{n-m}\left(M^{n} ; Z\right)$ is not finitely generated.

2. $H^{n-m}\left(M^{n} ; Z\right)=0$ and $M^{n} / \pi$ is open.

3. $H^{n-m}\left(M^{n} ; Z\right) \simeq Z$ or $Z_{2}$ and $M^{n} / \pi$ is closed.

Received by the editors December 12, 1957. 
It will be apparent that we need only consider $\pi$ to be free abelian of rank $m$. We shall assume that $H^{n-m}\left(M^{n} ; Z\right)$ is finitely generated, then for every prime $p$

$$
H^{n-m}\left(M^{n} ; Z_{p}\right) \simeq Z_{p} \otimes H^{n-m}\left(M^{n} ; Z\right)
$$

is a finite dimensional vector space over $Z_{p}$. The action of $\pi$ on $M^{n}$ induces a linear representation of $\pi$ on $H^{n-m}\left(M^{n} ; Z_{p}\right)$. Since $H^{n-m}\left(M^{n} ; Z_{p}\right)$ is finite dimensional, the kernel of this representation $\pi(p) \subset \pi$ is a free abelian subgroup of finite index in $\pi$, thus rank $(\pi(p))=m$. We may replace $\left(\pi, M^{n}\right)$ by $\left(\pi(p), M^{n}\right)$ and observe that $\pi(p)$ has a trivial representation on $H^{n-m}\left(M^{n} ; Z_{p}\right)$. The spectral sequence of the covering $\left(\pi(p), M^{n}\right)$ has an $E_{2}$-term of the form

$$
E_{2}^{m, n-m} \simeq H^{m}\left(\pi(p) ; Z_{p}\right) \otimes H^{n-m}\left(M^{n} ; Z_{p}\right) .
$$

By the maximal cocycle argument

$$
H^{n}\left(M^{n} / \pi(p) ; Z_{p}\right) \simeq E_{2}^{m, n-m} \simeq H^{n-m}\left(M^{n} ; Z_{p}\right) .
$$

It is clear that $M^{n} / \pi$ is compact if and only if $M^{n} / \pi(p)$ is compact. Thus, if $M^{n} / \pi$ is open, $H^{n}\left(M^{n} / \pi(p) ; Z_{p}\right)=0$ for every prime, so that $H^{n-m}\left(M^{n} ; Z\right)=0$. If $M^{n} / \pi$ is closed, then $H^{n-m}\left(M^{n} ; Z_{2}\right) \simeq Z_{2}$. In particular, if $M^{n} / \pi$ is closed, the rank of $H^{n-m}\left(M^{n} ; Z\right)$ is no greater than one. Such a group has a finite group of automorphisms so that the representation of $\pi$ on $H^{n-m}\left(M^{n} ; Z\right)$ has a kernel $\pi^{\prime} \subset \pi$ of rank $m$. If $M^{n} / \pi$ is closed, so is $M^{n} / \pi^{\prime}$, thus

$H^{m}\left(\pi^{\prime} ; Z\right) \otimes H^{n-m}\left(M^{n} ; Z\right) \simeq H^{n-m}\left(M^{n} ; Z\right) \simeq H^{n}\left(M^{n} / \pi^{\prime} ; Z\right) \simeq Z$ or $Z_{2}$.

This result can be extended to a manifold $B^{n}$ with boundary $b B^{n}$. For the relative cohomology groups $H^{i}\left(B^{n}, b B^{n} ; Z\right)$ the statement is unchanged. If the absolute groups $H^{i}\left(B^{n} ; Z\right)$ are used, then either $H^{n-m}\left(B^{n}, Z\right)$ is zero or it is not finitely generated. This result is related to a theorem of Hopf [3] and to theorems of Specker [5]. Smith [7] has given examples of a manifold with an abelian fundamental group whose rank exceeds the dimension of the manifold. Our result shows that in such a case the universal covering manifold must have a cohomology group that is not finitely generated.

We shall consider the action of a finitely generated solvable group of proper transformations. If $\pi$ is finitely generated and solvable, let $\pi=\pi^{(0)} \supset \pi^{(1)}, \cdots, \supset \pi^{(k)}=\{e\}$ be the derived series, then $\pi^{(i)} / \pi^{(i+1)}$ is a finitely generated abelian group and we define the rank of $\pi$ [4] to be 


$$
\operatorname{rank}(\pi)=\sum_{i=0}^{k} \operatorname{rank} \pi^{(i)} / \pi^{(i+1)} .
$$

LEMMA 1. Let $\left(\pi, M^{n}\right)$ be a finite group of proper transformations, and suppose there is an integer $j$ such that $H^{j}\left(M^{n} ; Z_{2}\right) \simeq Z_{2}$ and $H^{i}\left(M^{n} ; Z_{2}\right)=0, i>j$, then $H^{i}\left(M^{n} / \pi ; Z_{2}\right) \simeq Z_{2}$ and $H^{i}\left(M^{n} / \pi ; Z_{2}\right)=0$, $i>j$.

By duality, the hypothesis of the lemma implies that $H_{c}^{n-j}\left(M^{n} ; Z_{2}\right)$ $\simeq Z_{2}$ and $H_{c}^{i}\left(M^{n} ; Z_{2}\right)=0, i<n-j$. We consider the spectral sequence (2). The minimal base degree is 0 , and the minimal fibre degree is $n-j$; so by the minimal cocycle argument

$$
\hat{E}_{2}^{0, n-j} \simeq H^{0}\left(\pi ; Z_{2}\right) \simeq Z_{2} \simeq H_{c}^{n-j}\left(M^{n} / \pi ; Z_{2}\right)
$$

and $H_{c}^{i}\left(M^{n} / \pi ; Z_{2}\right)=0$ for $i<n-j$. We apply the duality theorem to $M^{n} / \pi$ to complete the argument.

THEOREM 2. Let $\left(\pi, M^{n}\right)$ denote the action of a finitely generated solvable group of rank $m$ as a proper group of transformations. If there is an integer $j$ such that $H^{j}\left(M^{n} ; Z_{2}\right) \simeq Z_{2}$ and $H^{i}\left(M^{n} ; Z_{2}\right)=0, i>j$, then $H^{n+m}\left(M^{n} / \pi ; Z_{2}\right) \simeq Z_{2}$ and $H^{i}\left(M^{n} / \pi ; Z_{2}\right)=0, i>j+m$.

The argument is based on induction over the rank. It is true for rank 0 , for then $\pi$ is finite. In the derived series $=\pi^{(0)} \supset \pi^{(1)}, \ldots$ choose the smallest value of $i$ for which the rank of $\pi^{(i)} / \pi^{(i+1)}$ is positive, so rank $\pi^{(i+1)}=m_{1}<m$, and rank of the abelian group $\pi^{(i)} / \pi^{(i+1)}$ is $m-m_{1}$. Let us consider $\left(\pi^{(i)} / \pi^{(i+1)}, M^{n} / \pi^{(i+1)}\right)$. By the induction hypothesis, $H^{j+m_{1}}\left(M^{n} / \pi^{(i+1)} ; Z_{2}\right) \simeq Z_{2}$, and $H^{i}\left(M^{n} / \pi^{(i+1)} ; Z_{2}\right)=0$, $i>j+m_{1}$. By Lemma 1 , we may consider $\pi^{(i)} / \pi^{(i+1)}$ as a free abelian group of rank $m-m_{1}$. We then apply the maximal cocycle argument to the spectral sequence (1) and conclude that $H^{j+m}\left(M^{n} / \pi^{(i)} ; Z_{2}\right) \simeq Z_{2}$ and $H^{i}\left(M^{n} / \pi^{(i)} ; Z_{2}\right)=0, i>j+m$. Using Lemma 1 again, we finally conclude that $H^{j+m}\left(M^{n} / \pi ; Z_{2}\right) \simeq Z_{2}$ and $H^{i}\left(M^{n} / \pi ; Z_{2}\right)=0, i>j+m$.

Now we state our extension of Mostow's result. Let $G$ and $H$ denote a simply connected Lie group together with a closed subgroup. It is well known that $G=L \times E^{s}, H^{c}=L^{\prime} \times E^{t}$, where $L$ and $L^{\prime}$ are maximal compact subgroups and $E^{s}$ and $E^{t}$ are Euclidean spaces [4]. By $H^{c}$ we denote the identity component of $H$.

Theorem 3. If $\pi_{1}(G / H)$ is solvable, then

$$
\text { rank } \pi_{1}(G / H) \leqq s-t
$$

and equality holds if and only if $G / H$ is compact.

The universal covering space of $G / H$ is $M^{n}=G / H^{c}$. Let $\pi=\pi_{1}(G / H)$, 
then we have the action of a finitely generated solvable group as a proper transformation group. The mapping

$$
\nu: G \rightarrow G / H^{c}
$$

is a fibration, so there is a spectral sequence with

$$
E_{2}^{p, q} \simeq H^{p}\left(M^{n} ; Z_{2}\right) \otimes H^{q}\left(H^{c} ; Z_{2}\right)
$$

and whose $E_{\infty}$-term is associated with $H^{*}\left(G ; Z_{2}\right)$. We return to the maximal cocycle argument again, so that

$$
\begin{aligned}
H^{n-8+t}\left(M^{n} ; Z_{2}\right) & \simeq Z_{2}, \\
H^{i}\left(M^{n} ; Z_{2}\right) & =0, \quad i>n-s+t .
\end{aligned}
$$

Since $M^{n} / \pi$ is $n$-dimensional, we apply Theorem 2 to conclude that

$$
\operatorname{rank}(\pi)+n-s+t \leqq n
$$

or

$$
\operatorname{rank}(\pi) \leqq s-t .
$$

A necessary and sufficient condition for the equality to hold is that $H^{n}\left(M^{n} / \pi ; Z_{2}\right) \simeq Z_{2}$, which is just the condition that $M^{n} / \pi=G / H$ be compact.

In a certain sense we can give an exact formula for the rank.

Corollary. If $G / H$ is a homogeneous n-dimensional space of $G$, which is a simple connected Lie group, and if $k$ is the largest integer such that $H^{k}\left(G / H ; Z_{2}\right) \neq 0$, then if $\pi_{1}(G / H)$ is solvable,

$$
\operatorname{rank}\left(\pi_{1}(G / H)\right)=(k-n)+(s-t) .
$$

This is an immediate corollary to Theorem 2 since $H^{n-s+t}\left(M^{n} ; Z_{2}\right)$ $\simeq Z_{2}$, and $H^{i}\left(M^{n} ; Z_{2}\right)=0, i>n-s+t$. We observe in closing that we have only used the most elementary properties of the spectral sequence of a covering. Perhaps refining the technique would lead to more information about homogeneous spaces with solvable fundamental group.

\section{REFERENCES}

1. A. Borel, Nouvelle demonstration d'un theoreme de P. A. Smith, Comment. Math. Helv. vol. 29 (1955) pp. 27-39.

2. H. Cartan and S. Eilenberg, Homological algebra, Princeton University Press, 1956.

3. H. Hopf, Enden offener Raume und undendliche diskontinvierlich Gruppen, Comment. Math. Helv. vol. 16 (1943) pp. 81-100. 
4. G. D. Mostow, On the fundamental group of a homogeneous space, Ann. of Math. (2) vol. 66 (1957) pp. 249-255.

5. E. Specker, Die erste Cohomologie gruppe von Uberlagerungen und Homotopieeigenschaften dreidimensionaler Mannigfaltigkeiten, Comment. Math. Helv. vol. 23 (1949) pp. 303-333.

6. P. A. Smith, The fundamental group of a group manifold, Ann. of Math. (2) vol. 36 (1935) pp. 210-229.

7. - Manifolds with abelian fundamental groups, Ann. of Math. (2) vol. 37 (1936) pp. 526-533.

UNIVERSITY OF Michigan

\section{ON THE GROUP OF AFFINITIES OF LOCALLY AFFINE SPACES}

\section{LOUIS AUSLANDER ${ }^{1}$}

Let $M$ be a compact manifold with a given complete flat affine connection (i.e., an affine connection with curvature and torsion zero). Then we may represent the fundamental group $\Gamma$ of $M$ by affine transformations of the real affine space $R^{n}$, in such a way that the orbit space of $R^{n}$ by $\Gamma$ is homeomorphic to $M$. We will denote the full group of affine transformations of $R^{n}$ by $A(n)$ and the orbit space of $R^{n}$ under $\Gamma$ by $R^{n} / \Gamma$. We represent the elements of $A(n)$ as matrices of the form

$$
\left(\frac{A}{0} \mid \frac{v}{1}\right)
$$

where $A$ is the (nonsingular) linear transformation part and $v$ is the translational part. Let $G$ be the group of all affinities of $M$, i.e., the group of all homeomorphisms of $M$ onto itself which preserve the given affine structure on $M$. Nomizu proved in [3] that $G$ is a Lie group. Let $G_{1}$ denote the identity component of $G$. It is the purpose of this note to prove that $G_{1}$ is a nilpotent Lie group.

Now it is well known that any map of $M$ into itself can be lifted to a map of $R^{n}$ into itself, uniquely up to covering transformations, i.e., up to elements of $\Gamma$. The maps in $G_{1}$ lift to affine transformations of $R^{n}$. It is clear that $G^{*}$, the identity component of the subgroup of $A(n)$ so obtained, projects back onto $G_{1}$ as a covering group. Further, since $g^{*} \Gamma g^{*-1}=\Gamma$, for all $g^{*} \in G^{*}$ and since $G^{*}$ is connected and $\Gamma$ discrete, it follows easily that $G^{*}$ and $\Gamma$ commute elementwise.

Received by the Editors January 4, 1957.

1 This paper was written while the author was a National Science Foundation Post Doctoral Fellow. 